\title{
Firefly luciferase inhibition: a widely neglected problem
}

\author{
Albert Braeuning
}

Received: 10 October 2014 / Accepted: 24 November 2014 / Published online: 2 December 2014

(C) Springer-Verlag Berlin Heidelberg 2014

Numerous research groups are engaged in the analysis of the interference of plant constituents with the growth and survival of cancer cells. Among others, the polyphenolic trans-stilbene resveratrol and various flavonoids are extensively studied in this context. These compounds are generally thought to act in a cytoprotective, anti-tumorigenic manner, probably via their antioxidant and anti-inflammatory properties or by their ability to enhance the cytotoxicity of anticancer drugs. The underlying molecular details are addressed by an increasing number of publications, often by tracing back alterations in biological endpoints, e.g., apoptosis, to a substance-dependent disturbance of distinct cellular signaling pathways and transcription factors. For a recent review of resveratrol effects on the activity of various transcription factors, see Whitlock and Baek (2012). Luciferase-based reporter assays are among the most frequently used experimental tools to analyze the activity of downstream transcription factors of interest.

Unfortunately, an important confounder of luciferase reporter assays is still neglected by most researchers in the field. As many other enzymes, Photinus pyralis-derived firefly luciferase is subject to inhibition by chemicals acting as competitive or non-competitive inhibitors of the protein's catalytic activity, as reviewed by Auld et al. (2008) and by Leitao and Esteves da Silva (2010). In a routine assay setup, direct inhibition of the reporter enzyme by a substance present in the cell lysate cannot be easily distinguished from an inhibition of reporter gene transcription, if proper controls are missing. This is of pivotal importance for the research related to nutritional effects on the

\section{A. Braeuning $(\square)$}

Department of Food Safety, Federal Institute for Risk

Assessment, Max-Dohrn-Str. 8-10, 10589 Berlin, Germany

e-mail: Albert.braeuning@bfr.bund.de growth and survival of tumor cells, since a number of plant substances and closely related chemical entities are potent luciferase inhibitors: Resveratrol (3,5,4'-trihydroxy-transstilbene) is a powerful inhibitor of firefly luciferase with an $\mathrm{IC}_{50}$ of approximately 1-2 $\mu \mathrm{M}$ (Bakhtiarova et al. 2006; Braeuning and Vetter 2012), as is the structurally related NFkB inhibitor (E)-2-fluoro-4'-methoxystilbene (Braeuning and Vetter 2012). Certain flavonoids, for example, the kinase inhibitor PD98,059 (2'-amino-3'-methoxyflavone) and the widely used aryl hydrocarbon receptor agonist $\beta$-naphthoflavone, also inhibit firefly luciferase at concentrations far below what is routinely used for in vitro assays (Auld et al. 2008; Vaas et al. 2014; Wang 2002).

It is quite difficult to find a paper in the literature where the possibility of luciferase enzyme inhibition has been explicitly taken into account by the authors. This does, however, not necessarily mean that all luciferase reporter assays conducted with cells treated with substances such as resveratrol must have yielded inconclusive results (nor do problems with a luciferase reporter assay invalidate the findings obtained with other experimental approaches which are presented in the same paper). A long time of incubation with resveratrol rather seems to reduce the problem of luciferase-inhibitory effects by resveratrol (Braeuning and Vetter 2012), most likely by conversion of resveratrol to less-active metabolites. This time dependency has only been demonstrated for a few cell lines (Braeuning and Vetter 2012) and will most probably differ substantially between individual lines. However, resveratrol is routinely used in concentrations of up to $100 \mu \mathrm{M}$ which does by far exceed the concentrations used for luciferase inhibition in the aforementioned study. It thus often remains uncertain whether a reduction in firefly luciferase reporter activity observed under resveratrol treatment is in fact caused by the reduced transactivation potential of the transcription 
factor of interest or whether it might be caused merely by inhibition of catalytic activity of firefly luciferase.

For some publications, it is possible to indirectly reason the absence of pronounced firefly luciferase inhibition by resveratrol. This applies, for example, if luciferase reporter systems for different signaling pathways were applied under identical conditions with one of the reporters being down, but another one being up-regulated by the compound (Kim et al. 2011), or if the observed down-regulation of reporter activity by resveratrol is antagonized by means of genetic (e.g., mutational inactivation of a certain transcription factor binding site in the genetic region driving the expression of the reporter gene) or pharmacological (e.g., treatment of cells with a modulator of the signaling pathway of interest) interference (Huang et al. 2012; Yu et al. 2013).

Unfortunately, not all publications contain this type of experimental approach. When applying the selection criteria (1) resveratrol incubation $\leq 24 \mathrm{~h}$, (2) reporter activity inhibition $\geq 50 \%$, (3) no up-regulation of another luciferase reporter under identical conditions, and (4) no detailed mechanistic approach (see the above paragraph), a number of recent papers contain conclusions based on luciferase reporter data which might have been corrupted by luciferase inhibition by resveratrol, since data do not allow to firmly exclude this possibility. For example, Ren et al. (2013) reported down-regulation of NFkB-driven firefly luciferase reporter activity by resveratrol, while the substance did not affect transcription factor abundance, nuclear translocation, and DNA binding affinity. The conclusion of $\mathrm{NF \kappa B}$ inhibition by resveratrol in the paper is solely based on diminished luciferase reporter activity in the presence of resveratrol, the latter finding possibly being due to direct reporter enzyme inhibition rather than interference with the NFKB transcription factor. Similarly, Shi et al. (2009) showed a reduction in PSA-driven reporter activity by resveratrol, which was observed in the absence of effects on androgen receptor abundance. Kim et al. (2010) tried to establish a link between diminished food intake of resveratrol-exposed mice and reduced expression of the orexigenic neuropeptides NPY and AgRP in vitro, based on the reduction in NPY- and AgRP-driven firefly luciferase reporter activities by resveratrol as sole experimental evidence, which, however, is questionable since the system might have been disturbed by inhibition of firefly luciferase.

There are different ways of avoiding the problems arising when combining luciferase-inhibiting substances such as resveratrol or certain flavonoids with reporter assays. Proper controls, mechanistic approaches, timing, and choice of substance concentration will reduce the risk of unintended disturbance. Optimized firefly luciferase variants are available which are resistant against many known enzyme inhibitors (Auld et al. 2009). First of all, however, it is crucial to increase awareness for this long-ignored issue.

\section{References}

Auld DS, Southall NT, Jadhav A et al (2008) Characterization of chemical libraries for luciferase inhibitory activity. J Med Chem 51(8):2372-2386. doi:10.1021/jm701302v

Auld DS, Zhang YQ, Southall NT et al (2009) A basis for reduced chemical library inhibition of firefly luciferase obtained from directed evolution. J Med Chem 52(5):1450-1458. doi:10.1021/jm8014525

Bakhtiarova A, Taslimi P, Elliman SJ et al (2006) Resveratrol inhibits firefly luciferase. Biochem Biophys Res Commun 351(2):481484. doi:10.1016/j.bbrc.2006.10.057

Braeuning A, Vetter S (2012) The nuclear factor $\kappa$ B inhibitor (E)-2fluoro-4'-methoxystilbene inhibits firefly luciferase. Biosci Rep 32(6):531-537. doi:10.1042/BSR20120043

Huang W, Shang WL, Wang HD, Wu WW, Hou SX (2012) Sirt1 overexpression protects murine osteoblasts against TNF- $\alpha$-induced injury in vitro by suppressing the NF- $\mathrm{KB}$ signaling pathway. Acta Pharmacol Sin 33(5):668-674. doi:10.1038/aps.2011.189

Kim SJ, Lee YH, Han MD, Mar W, Kim WK, Nam KW (2010) Resveratrol, purified from the stem of Vitis coignetiae Pulliat, inhibits food intake in C57BL/6J Mice. Arch Pharm Res 33(5):775780. doi:10.1007/s12272-010-0518-5

Kim MH, Yoo DS, Lee SY et al (2011) The TRIF/TBK1/IRF-3 activation pathway is the primary inhibitory target of resveratrol, contributing to its broad-spectrum anti-inflammatory effects. Pharmazie 66(4):293-300

Leitao JM, Esteves da Silva JC (2010) Firefly luciferase inhibition. J Photochem Photobiol, B 101(1):1-8. doi:10.1016/j.jphotob iol.2010.06.015

Ren Z, Wang L, Cui J et al (2013) Resveratrol inhibits NF- $\mathrm{B}$ signaling through suppression of p65 and ІкB kinase activities. Pharmazie 68(8):689-694

Shi WF, Leong M, Cho E et al (2009) Repressive effects of resveratrol on androgen receptor transcriptional activity. PLoS One 4(10):e7398. doi:10.1371/journal.pone.0007398

Vaas S, Kreft L, Schwarz M, Braeuning A (2014) Cooperation of structurally different aryl hydrocarbon receptor agonists and $\beta$-catenin in the regulation of CYP1A expression. Toxicology 325C:31-41. doi:10.1016/j.tox.2014.08.010

Wang TT (2002) Beta-naphthoflavone, an inducer of xenobiotic metabolizing enzymes, inhibits firefly luciferase activity. Anal Biochem 304(1):122-126. doi:10.1006/abio.2001.5562

Whitlock NC, Baek SJ (2012) The anticancer effects of resveratrol: modulation of transcription factors. Nutr Cancer 64(4):493-502. doi:10.1080/01635581.2012.667862

Yu YH, Chen HA, Chen PS et al (2013) MiR-520h-mediated FOXC2 regulation is critical for inhibition of lung cancer progression by resveratrol. Oncogene 32(4):431-443. doi:10.1038/onc.2012.74 